Urol. int. 1974;29:235

\title{
Urinary Incontinence in Myelomeningocele
}

\begin{tabular}{|l|l|l|}
\hline A.S. & Alexander S. & Cass \\
\hline B.S. & Bert S. & Spence \\
\hline
\end{tabular}

Urology Service, Gillette Children's Hospital, St. Paul, Minn.

\section{Key Words}

Child

Spina bifida

Bladder

Surgery

Incontinence

Author's address: Dr. A. S. Cass, Urology Service, Gillette Children's Hospital, St. Paul, Minn. (USA)

Abstract

In our study there were 81 (88\%) incontinent children out of 91 aged 5 years or older in the 174 children born with spina bifida cystica. The sex of the child, the presence of nonprogressive hydrocephalus and the vertebral level of the lesion did not significantly influence the incidence of incontinence. Detrusor activity was present in $89 \%$ of the children evaluated. Upper tract deterioration was present in $41-44 \%$ of the children with incontinence.

Conservative management with Credé expression or urethral catheter drainage did not reduce upper tract changes and was not always successful in controlling the incontinence. Intestinal conduit surgery was successful in reducing upper tract changes and in managing incontinence. This surgery did have a significant complication rate mainly stomal stenosis

but early diagnosis and treatment of the complications prevented deterioration in the upper urinary tracts. 